Review

\title{
Association between Fusobacterium nucleatum and colorectal cancer: Progress and future directions
}

\author{
Sheng Zhang ${ }^{1,2}$, Sanjun Cai ${ }^{1,2} \bowtie$, Yanlei Ma ${ }^{1,2} \bowtie$ \\ 1. Department of Colorectal Surgery, Fudan University Shanghai Cancer Center, Shanghai, 200032, China. \\ 2. Department of Oncology, Shanghai Medical College, Fudan University, Shanghai, 200032, China. \\ $\triangle$ Corresponding author: Dr. Yanlei Ma, Department of Colorectal Surgery, Fudan University Shanghai Cancer Center; Department of Oncology, Shanghai \\ Medical College, Fudan University, 270 Dong'an Road, Shanghai, 20032, China, Tel.: +86 21 64175590; Fax: +86 21 54175590, E-mail: yanleima@fudan.edu.cn and \\ Dr. Sanjun Cai, Department of Colorectal Surgery, Fudan University Shanghai Cancer Center; Department of Oncology, Shanghai Medical College, Fudan \\ University, 270 Dong'an Road, Shanghai, 20032, China, Tel.: +86 21 64175590; Fax: +86 21 54175590, E-mail: sanjuncai@hotmail.com \\ (C) Ivyspring International Publisher. This is an open access article distributed under the terms of the Creative Commons Attribution (CC BY-NC) license \\ (https://creativecommons.org/licenses/by-nc/4.0/). See http://ivyspring.com/terms for full terms and conditions.
}

Received: 2017.11.27; Accepted: 2018.02.06; Published: 2018.04.18

\begin{abstract}
The initiation and progression of colorectal cancer (CRC) involves genetic and epigenetic alterations influenced by dietary and environmental factors. Increasing evidence has linked the intestinal microbiota and colorectal cancer. More recently, Fusobacterium nucleatum (Fn), an opportunistic commensal anaerobe in the oral cavity, has been associated with CRC. Several research teams have reported an overabundance of $\mathrm{Fn}$ in human CRC and have elucidated the possible mechanisms by which $\mathrm{Fn}$ is involved in colorectal carcinogenesis in vitro and in mouse models. However, the mechanisms by which Fn promotes colorectal carcinogenesis remain unclear. To provide new perspectives for early diagnosis, the identification of high risk populations and treatment for colorectal cancer, this review will summarize the relative research progresses regarding the relationship between Fn and colorectal cancer.
\end{abstract}

Key words: Fusobacterium nucleatum; colorectal cancer; intestinal microbiota.

\section{Introduction}

Colorectal cancer (CRC) is the third most common malignancy in males and the second most common in females, with about 1.4 million new cases in 2012 that lead to 0.69 million deaths according to the World Health Organization[1]. Many risk factors have been identified for colorectal cancer, mainly including a family history of colorectal cancer, inflammatory bowel disease, excessive consumption of red meat, diabetes and obesity[2]. Recently, accumulating evidences has suggested that bacteria dysbiosis may be a risk factor for colorectal adenoma and cancer[3-7]. There is a huge colonization of the human intestine by the microbiome, containing more than 1000 different categories and $10^{14}$ microorganisms $[8,9]$. These microorganisms can play an important role in the maintenance of epithelial homeostasis and protection against potential pathogens[10, 11] Fusobacterium nucleatum (Fn) is an intestinal and oral microbe that has attracted increasing attention in the field of colorectal cancer[12-14]. $\quad F n$ is a gram-negative anaerobic bacterium that mainly resides in the human oral cavity and plays a significant role in the development of periodontal disease and gingivitis[15-17]. It is also involved in many infectious diseases outside of the oral cavity, such as brain abscesses, liver abscesses, and intrauterine infections associated with pregnancy complications[18-20]. Fn can adhere to and invade epithelial and endothelial cells via the adhesin FadA, which contributes to its dissemination in hosts[17, 21, 22]. Several studies have confirmed the enrichment of Fn in CRC $[5,12]$. Moreover, the abundance of Fn in inflammatory bowel disease is significantly higher than that in normal bowels and it becomes a risk factor for inflammation-associated colorectal cancer[23]. Fn may have great potential to promote colorectal cancer, analogous to the promotion of stomach cancer and MALT lymphoma by Helicobacter 
pylori $[24,25]$. However, it is unclear whether and how Fn promotes CRC progression.

\section{Clinical Significance of Fn Enrichment in CRC}

An enrichment of Fn in CRC tissues compared to the level in controls has been confirmed by many studies, but there are still many inconsistencies in other respects. Several studies demonstrated that the amount of tissue Fn DNA was positively associated with colorectal cancer-specific mortality, which suggested that the enrichment of Fn in CRC tissue might serve as a negative prognostic biomarker[12, 26-28]. A positive association between Fn abundance and lymph node metastasis has also been observed in CRC $[5,27,29]$. These findings may suggest Fn-high $\mathrm{CRC}$ as a more biologically aggressive cancer subtype. However, other researches did not observe the negative prognostic value of Fn in colorectal cancer[5, 30-32]. Therefore, it is still unclear whether Fn can be used as an independent prognostic factor for CRC. Fn is enriched in colonic adenoma and stool samples from patients with colorectal adenoma and carcinoma, and the abundance of Fn is consistent with the normal-adenoma-carcinoma sequence model, which suggests that Fn accumulation is an event that occurs at an early stage of colonic tumorigenesis[26, 33 , 34]. Notably, recent studies have demonstrated that Fn may also be involved in the serrated neoplasm pathway[30, 35], an alternative pathway of CRC development that contributes to premalignant colorectal lesions[36, 37]. As we know, identifying colorectal adenomas or early cancer by screening can effectively reduce colorectal cancer mortality[38, 39]. Traditional colonoscopy as an invasive examination method cannot be widely used in screening for colorectal neoplasia. In contrary, the detection of biomarkers in feces or blood has a broad potential application in screening for colorectal cancer[40, 41]. Interestingly, several studies have reported the potential value of $\mathrm{Fn}$ in improving the diagnosis of colorectal neoplasia. Wong $\mathrm{SH}$ et al reported that quantification of fecal Fn combined with fecal immunochemical test can improve the diagnosis of advanced colorectal adenoma and carcinoma[42]. In this study, the relative abundance of fecal Fn in CRC group was 132 times that of the control group, and was 3.8-fold higher in the advanced adenoma group than in the control group. Liang $Q$ et al found that with fecal Fn alone, the sensitivity and specificity of CRC diagnosis was $80.2 \%$ and $80.7 \%$, respectively, while the sensitivity increased to $92.8 \%$ when fecal Fn was used in combination with FIT and three other fecal bacteria[43]. A metagenomic profiling study of the CRC fecal microbiomes indicated that
butyryl-CoA dehydrogenase, a gene marker from Fn, can serve as a non-invasive and effective diagnostic biomarker of CRC[44]. Wang HF et al found that serum anti-Fn-IgA can serve as a potential biomarker for the early diagnosis of CRC[45].Collectively, with the detection of Fn, fecal and serum microbiome-based strategies might practical tools for the early diagnosis and treatment of colorectal adenoma and carcinoma.

\section{Location and Translocation Route of Fn in vivo}

How Fn is able to colonize the gut mucosa from the oral cavity is still unknown. A recent study reported that the microbial community types observed in the oral cavity and the gut were predictive of each other[46], which may suggest an oral-gut translocation route for Fn. Two possible routes might be involved in this process. The first possible route is via swallowing the bacteria, which may further lead to dysbiosis of the gut microbiota, disruption of gut homeostasis, and alteration of the microenvironment that favors the development of CRC[47]. In the $\mathrm{APC}^{\text {mint }}$ mouse model, daily administration of Fn can increase colon tumor load, and Fn can be isolated from tumors[14]. Fn is a leading cause of periodontal disease, which may increase the risk of CRC as reported by a retrospective analysis with a large samples size[48]. Interestingly, in another study, no association was found between Fn abundance in the oral cavity and colorectal cancer history[49]. Intravenously injected Fn can localize to mouse tumor tissues[50], and Fn was detected simultaneously in a pyrogenic liver abscess and colon cancer according to a case report[51]. Moreover, Fusobacterium can be detected in liver metastasis, and is stably and persistently associated with paired primary-metastatic CRC[52], which suggests that Fn might serve as part of the metastatic tissue colonization through a hematogenous route or the lymphatic metastasis pathway. However, different Fusobacterium isolated from colorectal tumor tissues may have different biological features and carry more potential virulence proteins compared to their oral counterparts[53]. Flanagan et al reported that Fn abundance in stool samples did not correlate with that in paired tissue samples[26]. Notably, Fn has been detected predominantly in proximal colon cancer, and the proportions of Fn-high CRC gradually increase from rectum to cecum[30, 54], this correlation between Fn prevalence and tumor location is consistent with the point of view that microbiota organization is a distinct feature of proximal CRC[55]. Accordingly, the exact mechanisms of dissemination from oral cavity to colon and the potential links 
between Fn abundance and tumor location remain to be further elucidated. Once known, those mechanisms will help us reveal the true role of Fn in colorectal carcinogenesis.

\section{Molecular Pathological Features in Fn-positive CRC}

Molecular pathological epidemiology, the concept proposed by Ogino and Stampfer, which deeply investigates the phenotypic outcomes of diseases such as cancer using molecular pathological analyses, may represent a new dimension and provide new insights into the carcinogenic mechanism of CRC[56-58].It is well-known that all that high microsatellite instability (MSI-H) is an important pathological characteristic of CRC[59]. Interestingly, many studies have observed a significant correlation between MSI-H and a greater amount of Fn in colorectal cancer tissues[30, 31, 60], although the underlying mechanism remains unknown. One possible explanation for this phenomenon is that reactive oxygen species(ROS) and inflammatory cytokines, induced by Fn in colorectal cancer tissues, can attenuate the activity of mismatch repair (MMR) protein leading to MSI-H[33, 61]. CpG island methylator phenotype (CIMP) is another characteristic feature of chronic inflammation and is closely related to carcinogenesis in many types of cancer. Several studies have reported that the presence of Fn is more often detected in high-CIMP CRC $[12,30,31,60]$. In addition, Fusobacterium-high CRC correlates with wild type TP53, mutant CHD7/8 and a high frequency of somatic mutation[60]. Therefore, Fusobacterium enrichment may be associated with specific molecular subsets of CRCs[62].Moreover, the combination of Fusobacterium detection and the molecular pathological features of CRC may provide us with insights into the role of microorganisms in carcinogenesis and better treatment options for patients based on molecular pathological epidemiology[56].

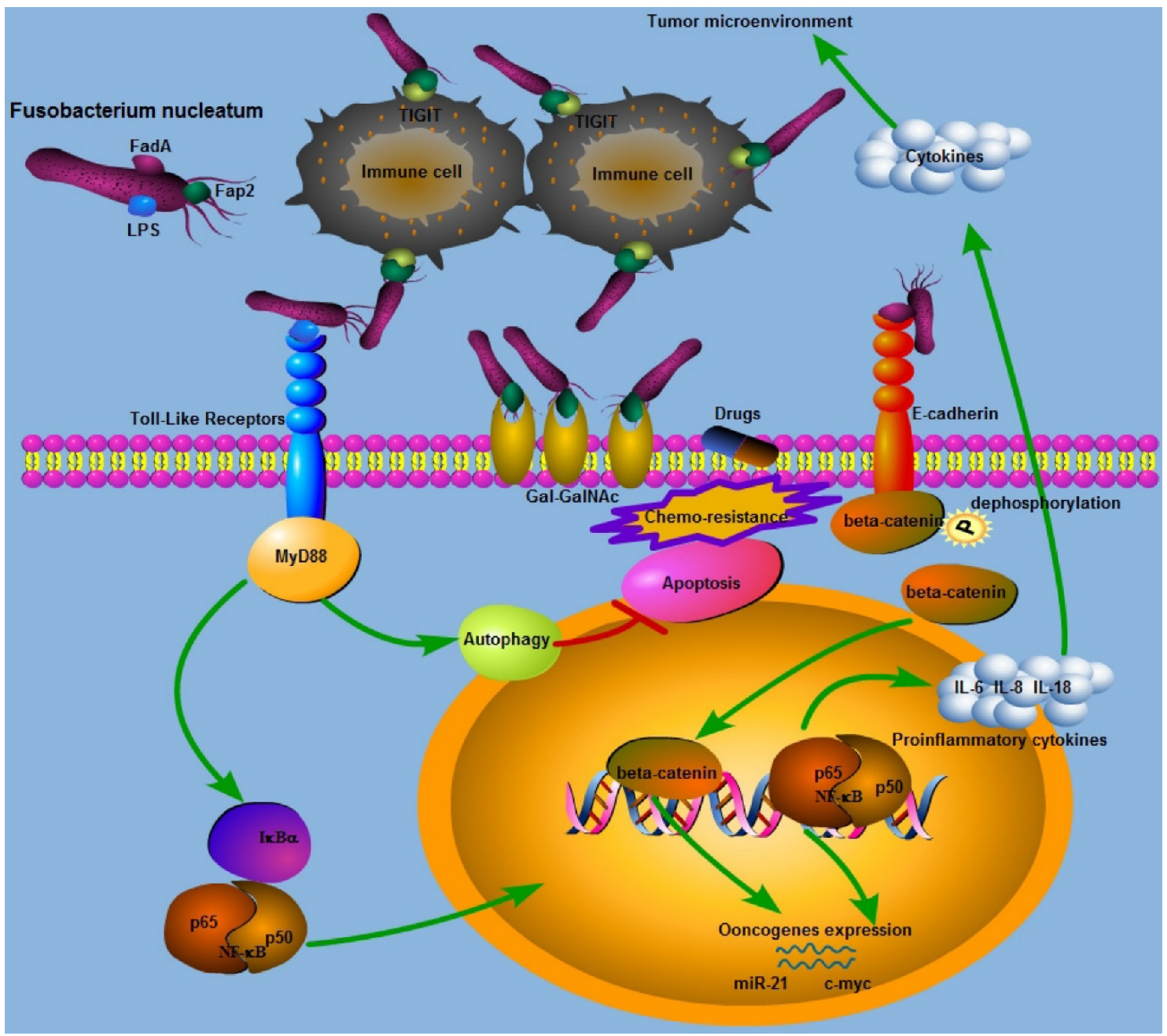

Figure 1. Schematic representation of the molecular mechanisms of Fn in colorectal cancer carcinogenesis and progression. 


\section{Diverse Detection of Fn in CRC}

The methods for detecting Fn include conventional quantitative PCR, metagenomic sequencing, Fluorescence in situ hybridization, and $16 S$ ribosomal RNA sequencing[63]. Among these detection methods, quantitative PCR is the least expensive and most widely used in large case studies. However, different sample types and different detection methods may result in ambiguous results. Therefore, there is an understandable inconsistency in the association between the Fn level and the clinicopathological features and prognosis of CRC among different studies. For example, sample types mainly include Formalin-fixed paraffin-embedded (FFPE) tissue blocks, fresh frozen tissues, stool, mucosal biopsies, and intestinal swabs. The rate of Fn-positive results in FFPE samples has been reported to be $8 \%$ in Japan and $13 \%$ in the United States[12, 32]. Mima et al found that the results of Fn detection in paired FFPE and frozen tissue specimens show a high linearity and high reproducibility, which suggests an acceptable level of detection of Fn in FFPE tissues[31]. Chen et al found that the microbial community of the intestinal lumen is different from that in the cancerous tissue, while the microbial community of cancerous tissue is similar to that in the adjacent noncancerous tissue[64]. In contrast, the Fn-positive rate is much higher in fresh frozen CRC tissues with the same method, quantitative PCR $[5,28,60]$. Therefore, the amount of Fn DNA detected in FFPE tissues might not precisely represent the actual abundance of Fn in CRC. However, another study indicated that the qPCR results were consistent when the abundance of Fn in gastroenterological cancer was detected in paired frozen and FFEP tissues[65]. Loop-mediated isothermal amplification primer sets that, detect two highly conserved genes in Fn, nusG and FadA, could serve as sensitive and rapid molecular tools for Fn detection [66].

\section{Is Fn a Driver or a Passenger?}

The role of Fn in CRC carcinogenesis remains uncertain. Whether Fn colonization is a consequence or a cause of CRC is unknown. A bacteria driver-passenger model proposed by Tjalsma et al may help us understand the complex mechanism of polymicrobial interaction in CRC[67]. In this model, 'driver' bacteria colonization contributes to epithelial DNA damage, creating an altered niche in the colon and leading to the exposure of epithelial cells to microbial compounds from 'passenger' bacteria. Then, 'driver' bacteria are gradually replaced by those 'passenger' bacteria, which can result in inflammation, barrier failure, and a change in the microenvironment that favors the development of CRC. In contrary, 'the alpha-bug' hypothesis proposed by Sears and Pardoll emphasizes the direct pro-oncogenic function of the major pathogenic bacteria, which can remodel the colonic bacteria community and modulate the tumor microenvironment[68]. It is plausible that the role of Fn may be as a 'driver' rather than a 'passenger' on the basis of the enrichment of Fn in colorectal adenoma. However, some studies have not supported this speculation. Flanagan et al found an over-abundance of Fn in cancerous tissue compared to that in normal tissue, while the Fn level in adenoma was not significantly higher than that in normal tissue[26]. Similarly, Amitay EL et al found that the abundance of Fn in feces was strongly associated with the presence of CRC, while no association was observed with the presence of colorectal adenoma, leading them to speculate that Fn was just a passenger rather than a driver in CRC development [69]. Interestingly, Tomkovich et al found that Fn did not exhibit proinflammatory or pro-tumorigenic activities in preclinical models of colon carcinogenesis, and these authors speculated that only a special group of Fn strains possess carcinogenic abilities when interacting with other members of the microbial community[70]. Accordingly, further investigation is needed into the molecular mechanism underlying the cross-talk between Fn and host proteins and, the structure and metabolic activities of the intestinal bacterial community, which may provide mechanistic insights into the relationship between Fn and colorectal tumor initiation and progression.

\section{Virulence Proteins of Fn Involved in CRC Carcinogenesis}

Although Fn has been associated with colorectal cancer (CRC), the underlying mechanisms remain unknown. Recently, two outer membrane proteins from Fn, Fap2 and FadA, have attracted attention. Fap2 is a galactose-sensitive hemagglutinin and adhesion protein, which contributes to the invasive ability of Fn[71]. Gur et al found that the Fap2 protein of Fn directly interacts with TIGIT expressed on NK cells and tumor-infiltrating lymphocytes, leading to the inhibition of NK cell cytotoxicity and lymphocytes activity[72]. Meanwhile, tumors infected with Fn were protected from NK-mediated killing and immune cell attack[72]. Abed et al identified a host polysaccharide, Gal-GalNAc, that is highly expressed in human CRC[50]. Moreover, Fap2 binding to Gal-GalNAc in human CRC mediates Fn enrichment in adenocarcinoma[50]. Interestingly, high levels of Gal-GalNAc has also been found in many other types of adenocarcinoma which may suggest that it is a 
potential target in the treatment of Fn-enriched cancers [73]. The FadA gene is unique to Fn, and the FadA protein, a surface adhesin expressed by Fn, plays an essential role in the process of cell attachment[21, 74]. Rubinstein et al demonstrated that FadA could bind to E-cadherin on CRC cells, mediating Fn attachment to and invasion of the epithelial cells. Binding of FadA to E-cadherin could further activate beta-catenin signaling, leading to elevated expression levels of oncogenes, Wnt genes, and inflammatory genes. In the above-mentioned process, clathrin mediated internalization is involved in activating the Wnt signaling pathway and promoting CRC carcinogenesis[75].Wnt pathway activation caused by APC gene mutation can affect the phosphorylation status of beta-catenin and initiate colorectal tumorigenesis [76-78]. However, another study has demonstrated that the presence of FadA and Fap2 adhesins in Fn was not sufficient to induce either inflammation or cancer in a preclinical mouse model of colon carcinogenesis[70].Toll-like receptor 4 (TLR4), an important receptor for bacteria lipopolysaccharide (LPS), is over-expressed in CRC and may also play a role in tumor promotion[79]. We have previously demonstrated that infection of CRC cells with Fn increases expression of miR-21 by activating TLR4 in a mouse model[80], and miR-21 could serve as a key inhibitor of colitis-associated colon cancer (CAC) [81]. Chen et al found that both Fn and LPS extracted from Fn could activate beta-catenin signaling in colorectal cancer via the TLR4/P-PAK1 cascade[82]. Collectively, the Wnt/beta-catenin signaling pathway appears to play an essential role in Fn mediated colorectal carcinogenesis, and more investigations into the three Fn virulence proteins is needed to further determine the mechanisms by which Fn promotes colorectal carcinogenesis.

\section{Fn, Inflammation and Immunity in CRC}

It is now well-established that chronic inflammation induced by bacterial infection increases the risk of cancer[83]. A recent study has provided insights into the relationship between the gut microbiome and inflammatory cytokine production capacity[84]. Fn infection induces local inflammation and increased expression of inflammatory cytokines, mainly including IL-6, IL-8, TNF- $\alpha$ and cox-2, in the tumor microenvironment, and these cytokines can promote tumorigenesis in CRC $[33,53,75,85,86]$. Ye et al found that Fn can induce the expression of the chemokine CCL20 in colorectal cancer cells when they are co-cultured with Fn[87]. Moreover, Fn can also stimulate monocyte/macrophage activation and migration which can promote colorectal cancer development $[87,88]$. NF- $\mathrm{kB}$ is a transcription factor that is involved in regulating the expression of many genes and that promotes tumor growth and progress $[89,90]$. NF- $\mathrm{KB}$ is more frequently activated in Fn-enriched CRC[33, 80, 86]. Consist with this finding, Rubinstein et al found that only wild-type Fn induced NF-kB expression in HCT116 xenografts[75]. As NF-kB serves as the central link between inflammation and cancer[91], it has been suggested that NF-kB activation may be involved in colorectal carcinogenesis in tumors with Fn enrichment. Kostic et al observed a proinflammatory expression phenomenon in CRC infected with Fn, and determined that Fn may expand myeloid-derived immune cell types, which inhibited the $\mathrm{T}$ cell-mediated immune response against colorectal tumors and facilitate tumor progression by modulating the tumor-immune microenvironment[33]. However, in that study, no association between Fn and inflammation-associated intestinal carcinogenesis was observed in the $\mathrm{APC}^{\mathrm{min}+}$ mouse model of intestinal tumorigenesis[33]. Fn can also display immunosuppressive activities by causing severe aggregation and apoptosis of immune cells[71, $88,92,93]$. In line with these experimental results, Mima et al observed an inverse association between the amount of Fn and CD3+ T cell density[31], while another study showed that high levels of Fn were not significantly associated with CD3+ T cell density[88]. We may infer the immunosuppressive role of Fn in colorectal cancer initiation or development. The gut microbiota has been reported to affect the response to anticancer immunotherapy including PD-L1 and CTLA-4[94, 95]. Based on this, whether Fn affects antitumor immune therapy is very promising route for further investigation, which may provide us with valuable insights into clinical management of CRC patients.

\section{Fn, Lifestyle and Drug Treatment in CRC}

It is reported that a 'western' lifestyle characterized by a high consumption of fat, processed meat and red meat, and a low intake of fiber can increase the risk of CRC through the promotion bacterial outgrowth that may lead to a hostile gut environment [96-99]. A prospective cohort study demonstrated that prudent diets rich in whole grains and dietary fiber are associated with a lower risk for Fn-positive CRC, which suggests a potential role of Fn in mediating the association between diet and colorectal cancer [100]. A recent study has shown that the Fn abundance in fecal samples was not associated with lifestyle or dietary habits[69]. In addition, different dietary patterns may lead to the variation in Fn abundance observed in different countries[32, 98, 101]. It has been reported that short-chain fatty acids 
(SCFA), gut microbiota-derived bacterial fermentation products, can promote colonic homeostasis and health[102]. Commensal gut microflora and probiotics may exert antimicrobial activity against CRC promoting bacteria by producing colonic fermentation products, such as SCFA and antimicrobial peptides, which can lower intestinal $\mathrm{pH}$, change the immune microenvironment, and eventually create a less hospitable environment for colonization by Fn[6, 103-105]. These findings may imply that Fn infection in CRC may be closely related to dietary and lifestyle exposure, two important subjects investigated by the evolving field of molecular pathological epidemiology[106]. Probiotics and prebiotics can increase the production of lactic acid bacteria by adjusting the balance of the intestinal microbiota, which can decrease the risk of CRC[107, 108]. A recent study showed that treatment of mice bearing a colon cancer xenograft with the antibiotic metronidazole can successfully decrease Fusobacterium load, cancer cell proliferation and tumor growth, suggesting antibiotics as a potential effective intervention for patients with Fn-positive CRC[52]. Guo et al found that a subunit of the antioxidant protein alkyl hydroperoxide reductase could serve as a potential vaccine candidate to protect against Fn infection, which may add a dimension to our strategy for the prevention of CRC associated with Fn infection[109]. Interestingly, bacterial metabolism can also differentially affect the host response to chemotherapeutic drugs including 5 -fluorouracil $(5-\mathrm{Fu})$ in the interspecies model system C. elegans $[110,111]$. Therefore, we may reason that the efficacy of 5-Fu, the principal anti-colorectal cancer drugs, may be affected by the intestinal microbiota. Consistent with these findings, $\mathrm{Yu}$ et al found that the amount of Fn was higher in a CRC recurrence group than that in a non-recurrence group among those who had received 5-Fu-based postoperative chemotherapy[112]. Moreover, Fn can mediate CRC chemoresistance in response to 5 -Fu by selectively targeting miR-18a* and miR-4802 followed by autophagy pathways activation, suggesting that Fn may be a potential risk factor for CRC recurrence[112]. Taken together, with dietary and lifestyle interventions and appropriate and specific drug treatment, targeting Fn may be a promising new way to address CRC associated with Fn infection, providing us with unique insights for future investigations to further develop our current clinical strategies for CRC.

\section{Future Direction and Concluding Remarks}

In this review, we focused on the potential association between $\mathrm{Fn}$ infection and colorectal carcinogenesis. The molecular mechanisms involved may include the WNT signaling pathway, the MSI pathway, the epithelial-to-mesenchymal transition (EMT) pathway[27], CIMP and the "serrated" pathway, a microRNA pathway and an inflammation pathway, which may help us to build special models linking the intestinal microbiota and CRC and to further understand how CRC initiates and progresses[113]. Although Fn infection is prevalent in human CRC, there is disagreement about the relationship between $\mathrm{Fn}$ and colorectal adenoma. Whether Fn colonization is a consequence or a cause of colorectal carcinogenesis remains unclear. While there is a significant correlation between MSI-H and overabundance of Fn in colorectal cancer tissues, the underlying mechanism remains unknown. Quantification of fecal Fn may serve as a promising biomarker for detecting colorectal neoplasm, although the prognostic value of Fn is controversial. Different sample types and diverse methods may affect the accuracy in detecting the abundance of Fn. FadA, Fap2, and LPS, as virulence proteins of Fn, may play essential roles in mediating Fn invasion and the promotion of CRC. Moreover, miRNA, autophagy and the WNT signaling pathway appear to be involved in Fn-mediated complex networks that promote colorectal cancer. Screening and identification of the virulence proteins of Fn may help us to further investigate the Fn-host cross-talk and to develop a new way to abrogate Fn infections[114, 115]. The immune suppression role and chemotherapy resistance mechanisms of Fn may alter our therapeutic and preventive strategies for CRC. We can now better understand the intestinal bacteria composition based on the technology of $16 \mathrm{~S}$ ribosomal DNA sequencing and metagenomic analysis. Nevertheless, we cannot yet establish an in vitro model that efficiently simulates the complex bacterial community and the colonization ecosystem. Thus, further investigation is needed to focus on the host-pathogen interaction partners and the virulence proteins that are involved in colorectal carcinogenesis.

\section{Competing Interests}

The authors have declared that no competing interest exists.

\section{References}

1. Torre LA, Bray F, Siegel RL, Ferlay J, Lortet-Tieulent J, Jemal A. Global cancer statistics, 2012. CA: a cancer journal for clinicians. 2015; 65: 87-108.

2. Brenner H, Kloor M, Pox CP. Colorectal cancer. Lancet (London, England). 2014; 383: 1490-1502.

3. Garcia-Castillo V, Sanhueza E, McNerney E, Onate SA, Garcia A. Microbiota dysbiosis: a new piece in the understanding of the carcinogenesis puzzle. J Med Microbiol. 2016; 65: 1347-1362.

4. Wu N, Yang X, Zhang R, Li J, Xiao X, Hu Y, et al. Dysbiosis signature of fecal microbiota in colorectal cancer patients. Microbial ecology. 2013; 66: 462-470. 
5. Castellarin M, Warren RL, Freeman JD, Dreolini L, Krzywinski M, Strauss J, et al. Fusobacterium nucleatum infection is prevalent in human colorectal carcinoma. Genome research. 2012; 22: 299-306.

6. Louis P, Hold GL, Flint HJ. The gut microbiota, bacterial metabolites and colorectal cancer. Nature reviews Microbiology. 2014; 12: 661-672.

7. Schwabe RF, Jobin C. The microbiome and cancer. Nat Rev Cancer. 2013; 13: $800-812$

8. Qin J, Li R, Raes J, Arumugam M, Burgdorf KS, Manichanh C, et al. A human gut microbial gene catalogue established by metagenomic sequencing. Nature. 2010; 464: 59-65.

9. Claesson MJ, Cusack S, O'Sullivan O, Greene-Diniz R, de Weerd H, Flannery E, et al. Composition, variability, and temporal stability of the intestinal microbiota of the elderly. Proc Natl Acad Sci U S A. 2011; 108 (Suppl 1): S4586-S4591.

10. Tremaroli V, Backhed F. Functional interactions between the gut microbiota and host metabolism. Nature. 2012; 489: 242-249.

11. Gill SR, Pop M, Deboy RT, Eckburg PB, Turnbaugh PJ, Samuel BS, et al. Metagenomic analysis of the human distal gut microbiome. Science. 2006; 312: 1355-1359.

12. Mima K, Nishihara R, Qian ZR, Cao Y, Sukawa Y, Nowak JA, et al. Fusobacterium nucleatum in colorectal carcinoma tissue and patient prognosis. Gut. 2016; 65: 1973-1980

13. Voigt AY, Zeller G, Bork P. Microbial Biomarkers for Early Cancer Detection. Dtsch Med Wochenschr. 2017: 142: 267-274.

14. Kostic Aleksandar D, Chun E, Robertson L, Glickman Jonathan N, Gallini Carey A, Michaud M, et al. Fusobacterium nucleatum Potentiates Intestinal Tumorigenesis and Modulates the Tumor-Immune Microenvironment. Cell Host \& Microbe. 2013; 14: 207-215.

15. Bolstad AI, Jensen HB, Bakken V. Taxonomy, biology, and periodontal aspects of Fusobacterium nucleatum. Clin Microbiol Rev. 1996; 9: 55-71.

16. Huttenhower C, Gevers D, Knight R, et al. The Human Microbiome Project Consortium; Structure, function and diversity of the healthy human microbiome. Nature. 2012; 486: 207-214.

17. Han YW, Shi W, Huang GT, Kinder Haake S, Park NH, Kuramitsu H, et al. Interactions between periodontal bacteria and human oral epithelial cells: Fusobacterium nucleatum adheres to and invades epithelial cells. Infect Immun. 2000; 68: 3140-3146.

18. Han YW, Fardini Y, Chen C, Iacampo KG, Peraino VA, Shamonki JM, et al. Term stillbirth caused by oral Fusobacterium nucleatum. Obstet Gynecol. 2010; 115: 442-445.

19. Kai A, Cooke F, Antoun N, Siddharthan C, Sule O. A rare presentation of ventriculitis and brain abscess caused by Fusobacterium nucleatum. J Med Microbiol. 2008; 57: 668-671.

20. Yoneda M, Kato S, Mawatari H, Kirikoshi H, Imajo K, Fujita K, et al. Liver abscess caused by periodontal bacterial infection with Fusobacterium necrophorum. Hepatol Res. 2011; 41: 194-196.

21. Xu M, Yamada M, Li M, Liu H, Chen SG, Han YW. FadA from Fusobacterium nucleatum utilizes both secreted and nonsecreted forms for functional oligomerization for attachment and invasion of host cells. The Journal of biological chemistry. 2007; 282: 25000-25009.

22. Fardini Y, Wang X, Temoin S, Nithianantham S, Lee D, Shoham M, et al. Fusobacterium nucleatum adhesin FadA binds vascular endothelial cadherin and alters endothelial integrity. Molecular microbiology. 2011; 82: 1468-1480.

23. Strauss J, Kaplan GG, Beck PL, Rioux K, Panaccione R, Devinney R, et al. Invasive potential of gut mucosa-derived Fusobacterium nucleatum positively correlates with IBD status of the host. Inflamm Bowel Dis. 2011; 17: 1971-1978.

24. Polk DB, Peek RM, Jr. Helicobacter pylori: gastric cancer and beyond. Nat Rev Cancer. 2010; 10: 403-414.

25. Cover TL, Blaser MJ. Helicobacter pylori in health and disease Gastroenterology. 2009; 136: 1863-1873.

26. Flanagan L, Schmid J, Ebert M, Soucek P, Kunicka T, Liska V, et al. Fusobacterium nucleatum associates with stages of colorectal neoplasia development, colorectal cancer and disease outcome. European journal of clinical microbiology \& infectious diseases : official publication of the European Society of Clinical Microbiology. 2014; 33: 1381-1390.

27. Yan X, Liu L, Li H, Qin H, Sun Z. Clinical significance of Fusobacterium nucleatum, epithelial-mesenchymal transition, and cancer stem cell markers in stage III/IV colorectal cancer patients. Onco Targets Ther. 2017; 10: 5031-5046.

28. Yamaoka $Y$, Suehiro $Y$, Hashimoto $S$, Hoshida T, Fujimoto $M$, Watanabe $M$, et al. Fusobacterium nucleatum as a prognostic marker of colorectal cancer in a Japanese population. J Gastroenterol 2017; [Epub ahead of print].

29. Li YY, Ge OX, Cao J, Zhou YJ, Du YL, Shen B, et al. Association of Fusobacterium nucleatum infection with colorectal cancer in Chinese patients. World J Gastroenterol. 2016; 22: 3227-3233.

30. Ito M, Kanno S, Nosho $K$, Sukawa $Y$, Mitsuhashi $K$, Kurihara $H$, et al. Association of Fusobacterium nucleatum with clinical and molecular features in colorectal serrated pathway. Int J Cancer. 2015; 137: 1258-1268.

31. Mima K, Sukawa Y, Nishihara R, Qian ZR, Yamauchi M, Inamura K, et al. Fusobacterium nucleatum and $\mathrm{T}$ Cells in Colorectal Carcinoma. JAMA oncology. 2015; 1: 653-661.

32. Nosho K, Sukawa Y, Adachi Y, Ito M, Mitsuhashi K, Kurihara H, et al. Association of Fusobacterium nucleatum with immunity and molecular alterations in colorectal cancer. World J Gastroenterol. 2016; 22: 557-566.
33. Kostic AD, Chun E, Robertson L, Glickman JN, Gallini CA, Michaud M, et al. Fusobacterium nucleatum potentiates intestinal tumorigenesis and modulates the tumor-immune microenvironment. Cell Host Microbe. 2013; 14: 207-215.

34. Fearon ER, Vogelstein B. A genetic model for colorectal tumorigenesis. Cell. 1990; 61: 759-767.

35. Park CH, Han DS, Oh YH, Lee AR, Lee YR, Eun CS. Role of Fusobacteria in the serrated pathway of colorectal carcinogenesis. Sci Rep. 2016; 6: 25271.

36. Rosty C, Hewett DG, Brown IS, Leggett BA, Whitehall VL. Serrated polyps of the large intestine: current understanding of diagnosis, pathogenesis, and clinical management. J Gastroenterol. 2013; 48: 287-302.

37. Bettington M, Walker N, Clouston A, Brown I, Leggett B, Whitehall V. The serrated pathway to colorectal carcinoma: current concepts and challenges. Histopathology. 2013; 62: 367-386.

38. Rex DK, Johnson DA, Anderson JC, Schoenfeld PS, Burke CA, Inadomi JM, et al. American College of Gastroenterology guidelines for colorectal cancer screening 2009. Am J Gastroenterol. 2009; 104: 739-750.

39. Sung JJ, Ng SC, Chan FK, Chiu HM, Kim HS, Matsuda T, et al. An updated Asia Pacific Consensus Recommendations on colorectal cancer screening. Gut. 2015; 64: 121-132

40. Xie YH, Gao QY, Cai GX, Sun XM, Zou TH, Chen HM, et al. Fecal Clostridium symbiosum for Noninvasive Detection of Early and Advanced Colorectal Cancer: Test and Validation Studies. EBioMedicine. 2017; 25: 32-40.

41. Eklof V, Lofgren-Burstrom A, Zingmark C, Edin S, Larsson P, Karling P, et al. Cancer-associated fecal microbial markers in colorectal cancer detection. Int J Cancer. 2017; 141: 2528-2536.

42. Wong SH, Kwong TNY, Chow TC, Luk AKC, Dai RZW, Nakatsu G, et al. Quantitation of faecal Fusobacterium improves faecal immunochemical test in detecting advanced colorectal neoplasia. Gut. 2017; 66: 1441-1448.

43. Liang Q, Chiu J, Chen Y, Huang Y, Higashimori A, Fang J, et al. Fecal Bacteria Act as Novel Biomarkers for Noninvasive Diagnosis of Colorectal Cancer. Clin Cancer Res. 2017; 23: 2061-2070.

44. Yu J, Feng Q, Wong SH, Zhang D, Liang QY, Qin Y, et al. Metagenomic analysis of faecal microbiome as a tool towards targeted non-invasive biomarkers for colorectal cancer. Gut. 2017; 66: 70-78.

45. Wang HF, Li LF, Guo SH, Zeng QY, Ning F, Liu WL, et al. Evaluation of antibody level against Fusobacterium nucleatum in the serological diagnosis of colorectal cancer. Sci Rep. 2016; 6: 33440.

46. Ding T, Schloss PD. Dynamics and associations of microbial community types across the human body. Nature. 2014; 509: 357-360.

47. Flynn KJ, Baxter NT, Schloss PD. Metabolic and Community Synergy of Oral Bacteria in Colorectal Cancer. mSphere. 2016; 1

48. Momen-Heravi F, Babic A, Tworoger SS, Zhang L, Wu K, Smith-Warner SA, et al. Periodontal disease, tooth loss and colorectal cancer risk: Results from the Nurses' Health Study. Int J Cancer. 2017; 140: 646-452.

49. Kato I, Vasquez AA, Moyerbrailean G, Land S, Sun J, Lin HS, et al. Oral microbiome and history of smoking and colorectal cancer. J Epidemiol Res. 2016; 2: 92-101.

50. Abed J, Emgard JE, Zamir G, Faroja M, Almogy G, Grenov A, et al. Fap2 Mediates Fusobacterium nucleatum Colorectal Adenocarcinoma Enrichment by Binding to Tumor-Expressed Gal-GalNAc. Cell Host Microbe. 2016; 20: 215-225.

51. Shigefuku R, Watanabe $T$, Kanno $Y$, Ikeda $H$, Nakano $H$, Hattori N, et al. Fusobacterium nucleatum detected simultaneously in a pyogenic liver abscess and advanced sigmoid colon cancer. Anaerobe. 2017; 48: 144-146.

52. Bullman S, Pedamallu CS, Sicinska E, Clancy TE, Zhang X, Cai D, et al. Analysis of Fusobacterium persistence and antibiotic response in colorectal cancer. Science. 2017; 358: 1443-1448.

53. Warren RL, Freeman DJ, Pleasance S, Watson P, Moore RA, Cochrane K, et al. Co-occurrence of anaerobic bacteria in colorectal carcinomas. Microbiome. 2013; 1 : 16

54. Mima K, Cao Y, Chan AT, Qian ZR, Nowak JA, Masugi Y, et al. Fusobacterium nucleatum in Colorectal Carcinoma Tissue According to Tumor Location. Clin Transl Gastroenterol. 2016; 7: e200.

55. Dejea CM, Wick EC, Hechenbleikner EM, White JR, Mark Welch JL, Rossetti BJ, et al. Microbiota organization is a distinct feature of proximal colorectal cancers. Proc Natl Acad Sci U S A. 2014; 111: 18321-18326.

56. Ogino S, Chan AT, Fuchs CS, Giovannucci E. Molecular pathological epidemiology of colorectal neoplasia: an emerging transdisciplinary and interdisciplinary field. Gut. 2011; 60:397-411.

57. Ogino S, Stampfer M. Lifestyle factors and microsatellite instability in colorectal cancer: the evolving field of molecular pathological epidemiology. J Natl Cancer Inst. 2010; 102: 365-367.

58. Ogino S, Nishihara R, VanderWeele TJ, Wang M, Nishi A, Lochhead P, et al. Review Article: The Role of Molecular Pathological Epidemiology in the Study of Neoplastic and Non-neoplastic Diseases in the Era of Precision Medicine. Epidemiology. 2016; 27: 602-611.

59. Boland CR, Goel A. Microsatellite instability in colorectal cancer. Gastroenterology. 2010; 138: 2073-2087.

60. Tahara T, Yamamoto E, Suzuki H, Maruyama R, Chung W, Garriga J, et al. Fusobacterium in colonic flora and molecular features of colorectal carcinoma. Cancer Res. 2014; 74: 1311-1318.

61. Schetter AJ, Heegaard NH, Harris CC. Inflammation and cancer: interweaving microRNA, free radical, cytokine and p53 pathways. Carcinogenesis. 2010; 31: $37-49$ 
62. Purcell RV, Visnovska M, Biggs PJ, Schmeier S, Frizelle FA. Distinct gut microbiome patterns associate with consensus molecular subtypes of colorectal cancer. Sci Rep. 2017; 7: 11590.

63. Drewes JL, White JR, Dejea CM, Fathi P, Iyadorai T, Vadivelu J, et al. High-resolution bacterial $16 \mathrm{~S}$ rRNA gene profile meta-analysis and biofilm status reveal common colorectal cancer consortia. NPJ Biofilms Microbiomes. 2017; 3: 34.

64. Chen W, Liu F, Ling Z, Tong X, Xiang C. Human intestinal lumen and mucosa-associated microbiota in patients with colorectal cancer. PLoS One. 2012; 7: 39743

65. Yamamura K, Baba Y, Miyake K, Nakamura K, Shigaki H, Mima K, et al. Fusobacterium nucleatum in gastroenterological cancer: Evaluation of measurement methods using quantitative polymerase chain reaction and a literature review. Oncol Lett. 2017; 14: 6373-6378.

66. Huang S, Yang Z, Zou D, Dong D, Liu A, Liu W, et al. Rapid detection of nusG and fadA in Fusobacterium nucleatum by loop-mediated isothermal amplification. J Med Microbiol. 2016; 65: 760-769.

67. Tjalsma H, Boleij A, Marchesi JR, Dutilh BE. A bacterial driver-passenger model for colorectal cancer: beyond the usual suspects. Nature reviews Microbiology. 2012; 10: 575-582.

68. Sears CL, Pardoll DM. Perspective: alpha-bugs, their microbial partners, and the link to colon cancer. J Infect Dis. 2011; 203: 306-311.

69. Amitay EL, Werner S, Vital M, Pieper DH, Hofler D, Gierse IJ, et al. Fusobacterium and colorectal cancer: causal factor or passenger? Results from a large colorectal cancer screening study. Carcinogenesis. 2017; 38: 781-788.

70. Tomkovich S, Yang Y, Winglee K, Gauthier J, Muhlbauer M, Sun X, et al. Locoregional Effects of Microbiota in a Preclinical Model of Colon Carcinogenesis. Cancer Res. 2017; 77: 2620-2632.

71. Coppenhagen-Glazer S, Sol A, Abed J, Naor R, Zhang X, Han YW, et al. Fap2 of Fusobacterium nucleatum is a galactose-inhibitable adhesin involved in coaggregation, cell adhesion, and preterm birth. Infect Immun. 2015; 83: 1104-1113.

72. Gur C, Ibrahim Y, Isaacson B, Yamin R, Abed J, Gamliel M, et al. Binding of the Fap2 protein of Fusobacterium nucleatum to human inhibitory receptor TIGIT protects tumors from immune cell attack. Immunity. 2015; 42: 344-355.

73. Abed J, Maalouf N, Parhi L, Chaushu S, Mandelboim O, Bachrach G. Tumor Targeting by Fusobacterium nucleatum: A Pilot Study and Future Perspectives. Front Cell Infect Microbiol. 2017; 7: 295.

74. Han YW, Ikegami A, Rajanna C, Kawsar HI, Zhou Y, Li M, et al. Identification and characterization of a novel adhesin unique to oral fusobacteria. J Bacteriol. 2005; 187: 5330-5340.

75. Rubinstein MR, Wang X, Liu W, Hao Y, Cai G, Han YW. Fusobacterium nucleatum promotes colorectal carcinogenesis by modulating E-cadherin/beta-catenin signaling via its FadA adhesin. Cell Host Microbe. 2013; 14: 195-206.

76. MacDonald BT, Tamai K, He X. Wnt/beta-catenin signaling: components, mechanisms, and diseases. Dev Cell. 2009; 17: 9-26.

77. Segditsas S, Tomlinson I. Colorectal cancer and genetic alterations in the Wnt pathway. Oncogene. 2006; 25: 7531-7537.

78. Pishvaian MJ, Byers SW. Biomarkers of WNT signaling. Cancer Biomark. 2007; 3: $263-2674$.

79. Santaolalla R, Sussman DA, Ruiz JR, Davies JM, Pastorini C, Espana CL, et al. TLR4 activates the beta-catenin pathway to cause intestinal neoplasia. PLoS One. 2013; 8: 63298.

80. Yang Y, Weng W, Peng J, Hong L, Yang L, Toiyama Y, et al. Fusobacterium nucleatum Increases Proliferation of Colorectal Cancer Cells and Tumor Development in Mice by Activating Toll-Like Receptor 4 Signaling to Nuclear Factor-kappaB, and Up-regulating Expression of MicroRNA-21. Gastroenterology. 2017; 152: 851-866.

81. Shi C, Yang Y, Xia Y, Okugawa Y, Yang J, Liang Y, et al. Novel evidence for an oncogenic role of microRNA-21 in colitis-associated colorectal cancer. Gut. 2016; 65: 1470-1481.

82. Chen Y, Peng Y, Yu J, Chen T, Wu Y, Shi L, et al. Invasive Fusobacterium nucleatum activates beta-catenin signaling in colorectal cancer via a TLR4/P-PAK1 cascade. Oncotarget. 2017; 8: 31802-31814.

83. de Martel C, Franceschi S. Infections and cancer: established associations and new hypotheses. Crit Rev Oncol Hematol. 2009; 70: 183-194.

84. Schirmer M, Smeekens SP, Vlamakis H, Jaeger M, Oosting M, Franzosa EA, et al. Linking the Human Gut Microbiome to Inflammatory Cytokine Production Capacity. Cell. 2016; 167: 1125-1136.

85. Dharmani P, Strauss J, Ambrose C, Allen-Vercoe E, Chadee K. Fusobacterium nucleatum infection of colonic cells stimulates MUC2 mucin and tumor necrosis factor alpha. Infect Immun. 2011; 79: 2597-2607.

86. Wei Z, Cao S, Liu S, Yao Z, Sun T, Li Y, et al. Could gut microbiota serve as prognostic biomarker associated with colorectal cancer patients' survival? A pilot study on relevant mechanism. Oncotarget. 2016; 7: 46158-46172.

87. Ye X, Wang R, Bhattacharya R, Boulbes DR, Fan F, Xia L, et al. Fusobacterium Nucleatum Subspecies Animalis Influences Proinflammatory Cytokine Expression and Monocyte Activation in Human Colorectal Tumors. Cancer Prev Res (Phila). 2017; 10: 398-409.

88. Park HE, Kim JH, Cho NY, Lee HS, Kang GH. Intratumoral Fusobacterium nucleatum abundance correlates with macrophage infiltration and CDKN2A methylation in microsatellite-unstable colorectal carcinoma. Virchows Arch. 2017; 471: 329-336
89. Inoue J, Gohda J, Akiyama T, Semba K. NF-kappaB activation in development and progression of cancer. Cancer Sci. 2007; 98: 268-274

90. Karin M. Nuclear factor-kappaB in cancer development and progression. Nature. 2006; 441: 431-436.

91. DiDonato JA, Mercurio F, Karin M. NF-kappaB and the link between inflammation and cancer. Immunol Rev. 2012; 246: 379-400.

92. Shenker BJ, DiRienzo JM. Suppression of human peripheral blood lymphocytes by Fusobacterium nucleatum. J Immunol. 1984; 132: 2357-2362.

93. Kaplan CW, Ma X, Paranjpe A, Jewett A, Lux R, Kinder-Haake S, et al. Fusobacterium nucleatum outer membrane proteins Fap2 and RadD induce cell death in human lymphocytes. Infect Immun. 2010; 78: 4773-4778.

94. Vetizou M, Pitt JM, Daillere R, Lepage P, Waldschmitt N, Flament C, et al. Anticancer immunotherapy by CTLA-4 blockade relies on the gut microbiota. Science. 2015; 350: 1079-1084

95. Sivan A, Corrales L, Hubert N, Williams JB, Aquino-Michaels K, Earley ZM, et al. Commensal Bifidobacterium promotes antitumor immunity and facilitates anti-PD-L1 efficacy. Science. 2015; 350: 1084-1089.

96. Watson AJ, Collins PD. Colon cancer: a civilization disorder. Dig Dis. 2011; 29: 222-228.

97. Song M, Garrett WS, Chan AT. Nutrients, foods, and colorectal cancer prevention. Gastroenterology. 2015; 148: 1244-1260

98. O'Keefe SJ, Li JV, Lahti L, Ou J, Carbonero F, Mohammed K, et al. Fat, fibre and cancer risk in African Americans and rural Africans. Nat Commun. 2015; 6: 6342 .

99. Feng Q, Liang S, Jia H, Stadlmayr A, Tang L, Lan Z, et al. Gut microbiome development along the colorectal adenoma-carcinoma sequence. Nat Commun. 2015; 6: 6528.

100. Mehta RS, Nishihara R, Cao Y, Song M, Mima K, Oian ZR, et al. Association of Dietary Patterns With Risk of Colorectal Cancer Subtypes Classified by Fusobacterium nucleatum in Tumor Tissue. JAMA oncology. 2017; 3: 921-927.

101. Kostic AD, Gevers D, Pedamallu CS, Michaud M, Duke F, Earl AM, et al. Genomic analysis identifies association of Fusobacterium with colorectal carcinoma. Genome research. 2012; 22: 292-298.

102. Smith PM, Howitt MR, Panikov N, Michaud M, Gallini CA, Bohlooly YM, et al. The microbial metabolites, short-chain fatty acids, regulate colonic Treg cell homeostasis. Science. 2013; 341: 569-573.

103. Ostaff MJ, Stange EF, Wehkamp J. Antimicrobial peptides and gut microbiota in homeostasis and pathology. EMBO Mol Med. 2013; 5: 1465-1483.

104. Roy CC, Kien CL, Bouthillier L, Levy E. Short-chain fatty acids: ready for prime time? Nutr Clin Pract. 2006; 21:351-366.

105. Canani RB, Costanzo MD, Leone L, Pedata M, Meli R, Calignano A. Potential beneficial effects of butyrate in intestinal and extraintestinal diseases. World J Gastroenterol. 2011; 17: 1519-1528.

106. Rescigno T, Micolucci L, Tecce MF, Capasso A. Bioactive Nutrients and Nutrigenomics in Age-Related Diseases. Molecules. 2017; 22: 105.

107. Coleman OI, Nunes T. Role of the Microbiota in Colorectal Cancer: Updates on Microbial Associations and Therapeutic Implications. Biores Open Access. 2016; 5: 279-288.

108. Ambalam P, Raman M, Purama RK, Doble M. Probiotics, prebiotics and colorectal cancer prevention. Best Pract Res Clin Gastroenterol. 2016; 30: 119-131.

109. Guo SH, Wang HF, Nian ZG, Wang YD, Zeng QY, Zhang G. Immunization with alkyl hydroperoxide reductase subunit $C$ reduces Fusobacterium nucleatum load in the intestinal tract. Sci Rep. 2017; 7: 10566.

110. Scott TA, Quintaneiro LM, Norvaisas P, Lui PP, Wilson MP, Leung KY, et al. Host-Microbe Co-metabolism Dictates Cancer Drug Efficacy in C. elegans. Cell. 2017; 169: 442-456.

111. Garcia-Gonzalez AP, Ritter AD, Shrestha S, Andersen EC, Yilmaz LS, Walhout AJM. Bacterial Metabolism Affects the C. elegans Response to Cancer Chemotherapeutics. Cell. 2017; 169: 431-441.

112. Yu T, Guo F, Yu Y, Sun T, Ma D, Han J, et al. Fusobacterium nucleatum Promotes Chemoresistance to Colorectal Cancer by Modulating Autophagy. Cell. 2017; 170: 548-563.

113. Colussi D, Brandi G, Bazzoli F, Ricciardiello L. Molecular pathways involved in colorectal cancer: implications for disease behavior and prevention. Int J Mol Sci. 2013; 14: 16365-16385.

114. Zanzoni A, Spinelli L, Braham S, Brun C. Perturbed human sub-networks by Fusobacterium nucleatum candidate virulence proteins. Microbiome. 2017; 5: 89

115. Kumar A, Thotakura PL, Tiwary BK, Krishna R. Target identification in Fusobacterium nucleatum by subtractive genomics approach and enrichment analysis of host-pathogen protein-protein interactions. BMC Microbiol. 2016; 16: 84 